\title{
Implementing the South African water policy: Holding the vision while exploring an uncharted mountain
}

\author{
HM MacKay ${ }^{1 *}$, KH Rogers ${ }^{1}$ and DJ Roux ${ }^{2}$ \\ ${ }^{1}$ Centre for Water in the Environment, University of the Witwatersrand, Private Bag 3, Wits 2050, South Africa \\ ${ }^{2}$ CSIR Environmentek, PO Box 395, Pretoria 0001, South Africa
}

\begin{abstract}
This paper discusses the long-term implementation of the South African National Water Policy of 1997, and addresses some of the difficult issues of the management and leadership of large change processes. Although the vision established by the water policy is clear, actually achieving that vision on the ground will require a robust, flexible, long-term implementation plan that is supported by all the role players responsible for water management in South Africa: Government, water services authorities, water services providers such as water boards, catchment management agencies (CMAs), water user associations, research organisations and the private sector. In this paper, we advocate a strategic, adaptive approach to policy implementation, which equates to "learn-bydoing", to meet the challenge of maintaining sufficient forward momentum in policy implementation, while still making sound decisions that take account of technical, environmental, economic, social and political factors.
\end{abstract}

Keywords: water policy, South Africa, adaptive management

\section{Introduction}

The South African water policy (DWAF, 1997) sets out a farreaching vision, making provision for the use of quite sophisticated policy tools for working towards sustainable, equitable and efficient water resources management. The vision for water management in South Africa is the product of radical changes in the social, political and water policy environments. It is considered internationally to be progressive, forward-thinking and ambitious. The vision is challenging, but has engendered considerable pride in those who helped develop it and those who are working to implement it.

Achieving this vision will require dramatic changes in the way in which water resource managers conduct their everyday business. We will need new institutions, new tools, a new mindset and a robust implementation plan to implement the water policy over the next 20 years and achieve its fully mature form. The implementation plan needs to set out the necessary steps, schedule and resources to be deployed. A wish list itemising all the nice things we wish to have or to be in so many years' time is not the same thing and will not achieve the desired outcomes.

The challenge facing the water sector in South Africa is daunting in its magnitude, and we have only just begun to take the first few steps of implementation. While the vision itself is explicit and attractive, clearly showing us what we would like to achieve, the big question remains: how are we going to achieve this vision? With limited human resources, limited finances, limited expertise and most of all, limited water resources, how will we move from the old to the new over the decades to come? What kind of implementation process will generate the necessary change and take us closer to the vision set out in the 1997 water policy, which has been paraphrased as "Some, for all, forever"?

\footnotetext{
* To whom all correspondence should be addressed.

Present address: Water Research Commission, Private Bag X03, Gezina 0031.

푱 +2712 330 0340; fax: +2712 331 2565; e-mail: heatherm@wrc.org.za Received 17 July 2002; accepted in revised form 27 June 2003.
}

\section{The change cycle}

The degree of change that is required to move from previous water policy to this new policy is significant. Change is always difficult - for a major change initiative to succeed it has to be carefully managed and strongly led. Change initiatives tend to pass through a typical cycle (Fig. 1): the early stages are characterised by doubt, fear and resistance, which need to be overcome by strong, visionary leadership and a careful, stepwise approach to implementation. At some point in the cycle, if the change initiative is to succeed, the promoters of the change have to ensure that the danger zone is passed; that a critical mass of people becomes positive and enthusiastic about the change and wholly espouses the new vision, in order for sufficient momentum to be built to carry the change through. Failure to build this critical mass and pass through the danger zone will result in reversal and a fall-back into old ways of doing business. Although a group is strongly influenced, negatively or positively, by the position of the group's leader or leadership, the position of an organisation or group in the change cycle tends to be the average of the individual positions within the organisation or group. This makes it imperative to deal with people as individuals, and to recognise the need to effect behavioural changes in individuals in order to advance the organisation's position.

In the case of water policy implementation, the group which will implement the policy includes the lead agent, the Department of Water Affairs and Forestry (DWAF), as well as several supporting organisations and institutions, including catchment management agencies (CMAs), water user associations, water services institutions and the private sector. This collaboration amongst many organisations to form the institutional framework for water policy implementation makes managing the change cycle even more complex, since one is no longer dealing with the issue of change within a single organisation.

\section{An adaptive approach to policy implementation}

Implementation of any major change initiative, such as the new water policy, is best effected by a stepwise, adaptive process which 


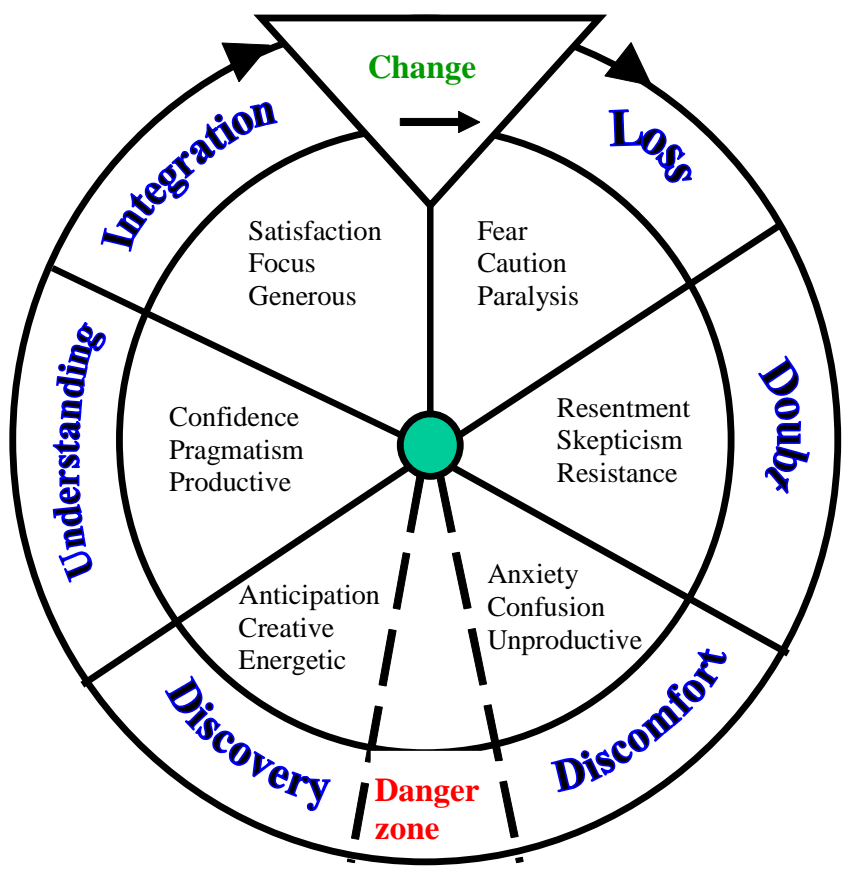

Figure 1

The cycle of organisational change (Brock \& Salerno, 1998)

allows for learning to be gathered along the way and fed back into improving the process. One benefit of this approach is that each step results in anchoring of incremental change in the institutional environment, so there is less likely to be a fall-back to the original situation. Implementing the new policy in a stepwise manner can be likened to climbing a mountain - although impatience might tempt us to reach for the top of the mountain in one "big jump", this is likely to be followed by a "big fall" back to the bottom. Installing new processes, new systems and new ways of thinking overnight, or in a very short time frame, leaves them inherently unstable and vulnerable to collapse. Climbing the mountain one stage at a time, establishing a camp as a firm anchor at each stage and moving forward in small steps, exploring from a stage camp, ensures slow but steady upward progress, and promotes safety since the degree of fall-back is limited to the previously established stage camp.

The mountaineering metaphor is apt, since implementation of the new water policy requires many of the same life skills: we have a vision of the top of the mountain that we want to climb, but the route is uncharted. No maps exist, and there are no easy welltravelled routes since the scope and breadth of the South African water policy's vision are unique in the world. If we proceed according to sound mountaineering technique, we will move gradually, establishing stage camps and making short exploratory expeditions from each stage camp, working with prototype tools until we fully understand the territory ahead. We must expect some failures - some routes from a camp will turn out to be dead-ends and we must learn from these. There will be no free lunches in the form of instructions for easy routes, although we can stay open to learning from others who may have tried out similar routes or similar mountains and have the skills and experience to know which routes might be less likely to fail.

Sustainable management of water resources is a journey without an end. We cannot stop managing once we have reached a comfortable position. New challenges and changes will face us all the time, as the political, social, economic and ecological environments around us change. We, in this generation, are tackling only the first mountain in a range: once we reach the top of this peak, we will see more mountains for the future. Even if we wished to, we could not hand over a fully achieved goal to the next generation, for them to sit back and reap the benefits. The best we can hand over is a sound process for climbing mountains, and the tools to climb the mountains that lie ahead.

What would an adaptive policy implementation process look like, and what principles should be followed in its application? If we continue the mountaineering metaphor, we can uncover a set of "mountaineering rules" to guide the process.

Mountaineering Rule 1: Pick your mountain, and try to get as good a view of the summit as possible. In our case, the water policy which we have developed and chosen to implement is our "mountain", and the vision of "Some for all forever" is the clearest view of the summit yet provided.

Mountaineering Rule 2: Pick your team, and make sure you include the right blend of skills and experience. Adaptive policy implementation is a process that incorporates multi-skilled teams, each member of which has a clear role to play in scaling the mountain - the expedition leader, the map-maker, the camp cook, specialist climbers who can deal with ice, rock or snow, loyal supporters who keep reminding the team of the vision, a camp manager to ensure smooth running of each camp on a daily basis even while all the climbers are out scouting on the mountain, reliable porters, and creative trouble-shooters. In water resource management, the necessary blend of expertise cannot be provided by DWAF alone, no matter how high the level of enthusiasm. Experts from the private sector, the academic sector and other government agencies will be needed on the team.

Mountaineering Rule 3: Take it step by step: don't go for the "big jump" approach. The process requires the establishment of safe stage camps, from which small proto-teams make short exploratory expeditions to scout the terrain, endeavouring to find the right direction for the next stage camp by trying a few different directions first, expecting some to fail. Taking small steps and expecting possible failure is very difficult in the public sector. However, a great deal of security and credibility can be achieved if the implementing agency is open and explicit about the short-term goals it sets, the actions to be taken towards these goals and the learning that occurs as goals are achieved, missed or revised. See also Rule 4.

Mountaineering Rule 4: Communicate, communicate, communicate. Lines of communication between team members must be kept open, and various forms of communication are used depending on the weather. In good conditions, where visibility is adequate, verbal communication may be sufficient to keep the teams together; in stormy weather, they must be roped and move very slowly forward. There must be trust and dialogue between all members of the team, for each relies on all the other members for the overall success of the expedition. It would not work if the leader simply issued orders from base camp. Maintaining communication across the full breadth of the water resources management "team" needs to be an explicit function of the policy implementation, and have its own strategy and goals.

Mountaineering Rule 5: Learn from exploration before committing to a particular route. Each evening the proto-teams gather back at the stage camp to discuss what they have learned, reflect on the best options and make decisions regarding the next phase of exploration. Advice from experts and experienced travellers plays a role in these decisions, as does the judgement of the expedition 
leader. Strong leadership is needed to bring the proto-teams together on a regular basis and send them out again in new directions. A learning process balances looking at the vision (the summit) with looking at the immediate next steps, and this balance requires careful management. Taking the time to be explicit about what a team has learned is not easily achieved when team leaders and team members are being pressed to deliver results, but not doing it is a recipe for mistakes, re-inventing of wheels and fall-back on the change cycle. On the other hand, taking short periods to affirm achievements generates confidence and enthusiasm.

\section{Basic road map of an adaptive process}

To follow a more conventional explanation, there is a cycle of learning and adapting that takes place as the expedition makes its way towards their vision, the summit of the mountain. There is a growing body of knowledge from the fields of ecosystem management (e.g. Aley et al., 1999; Rogers and Bestbier, 1997; Walters, 1997) and business management (Pieters, 1999; Roling and Wagemakers, 1998) which can demonstrate how an inherently ordered process of learning and adapting can be uncovered and applied with understanding to guide those responsible for the protection, control, management and allocation of natural resources such as water. When applied in ecosystem management, the process is considered as "learn-bydoing" and provides a way to ensure proactivity even in the face of uncertain consequences and future. In business, the approach is used to create organisations which can rapidly adjust to changing circumstances by making learning an explicit step in everything that they do (Fulmer, 2000; Haeckel, 1999).

A simple model has been derived from this body of knowledge (Fig. 2). The model presents a series of basic steps which can be applied in water resource management or any other form of management. Each of these steps and the linkages between them are described, along with some examples drawn from recent South African experiences, which apply particularly to the aquatic ecosystem protection aspects of the water policy.

\section{Step 1: Learn about the range of possible futures open to us}

This step requires us to gather knowledge about the possible futures open to us, depending on where we are now and what kind of trajectory we are presently on. Focusing on the future helps us to build consensus on a shared future, rather than becoming mired in conflicts over the current situation. Learning about the future may involve collection of new data or the synthesis of existing data in order to clearly establish the present situation and the trajectory. Thereafter comes the building of scenarios to represent or illustrate possible futures based on our present status and trajectory.

The water resource equivalent is the selection and consideration of a range of possible management classes that could be assigned to a water resource (such as a river), depending on its present state, trajectory, and desired future protection status. The water resource classification system, prescribed by law and cur- rently under development, will provide clear rules which apply in each management class, and which can be applied to maintain or move the water resource to a situation where the desired level of protection is being achieved.

The mountaineering equivalent is the discussion at base camp, advised by experts, of possible locations for the next stage camp and routes that might be taken to reach each of those possible locations. Imagine the chance of success if the team was not explicit about this process of researching where the potential next steps should lead and how to get there.

\section{Step 2: Decide which direction to move in}

Not all possible futures may be desirable to society. We need to take a decision on which futures we will discard as options for us, and which futures are desirable or consistent with the values on which our society is based. This decision must usually be taken in an environment of wide debate, consultation and participation from all sectors of civil society. The learning generated in Step 1 is used to inform the decision, so that people can understand the implications surrounding their choice of possible futures, and feel confident in their decision. The water resource equivalent of this step is the decision on the class which will be assigned to the water resource.

It is important to note that it is not usually possible to specify the exact future, its characteristics and appearance, in great detail (it's hard to clearly see the summit of a mountain from the bottom). Rather we may select a general direction in which we would like to move, which will take us towards a sub-set of possible endpoints, though these endpoints may share the same general values and characteristics (the water resource classes themselves are quite broad bands, translating for example into ranges for instream water quality, rather than single fixed concentrations). Even in moving towards the chosen subset of endpoints, there may be several different trajectories that we could follow. Frequently we can only map out the first few steps of a trajectory in any detail, and the path becomes more fuzzy as we look further along it.

There is a useful analogy from ecological theory which can illustrate this idea (Fig. 3). From the same starting point in the present, multiple trajectories with differing characteristics can take an ecosystem towards the same set of possible endpoints. An 


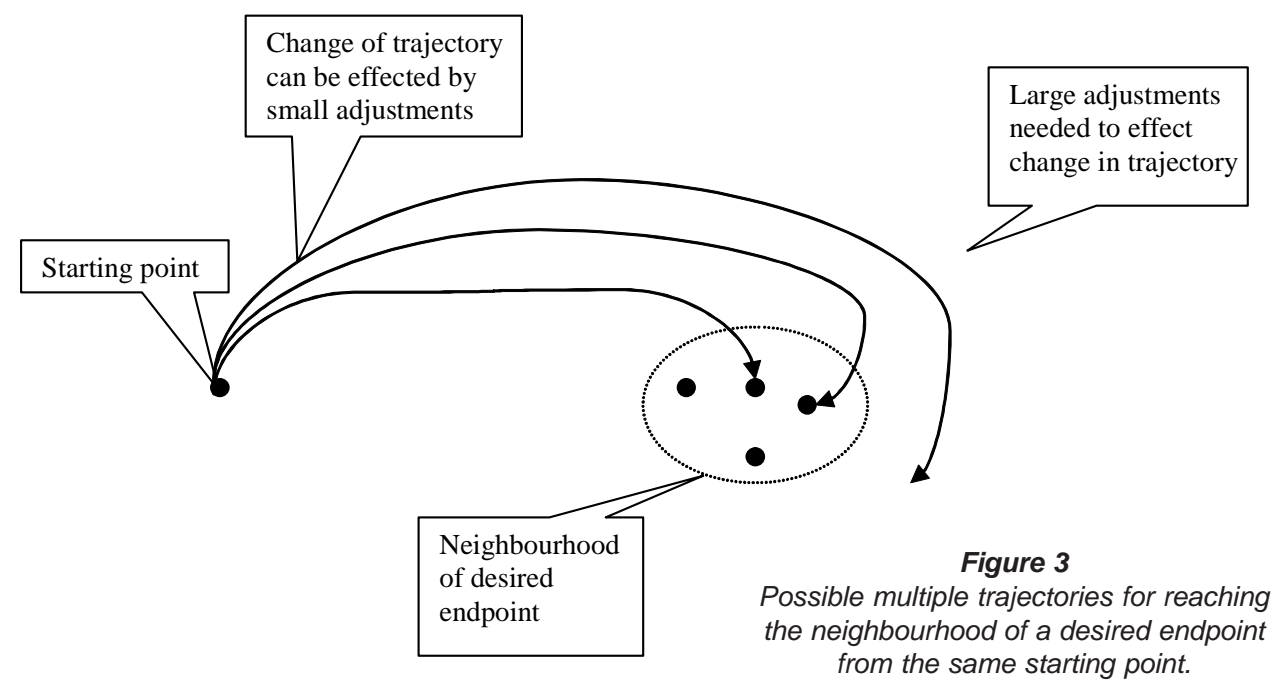

need to do this in an adaptive manner that ensures achievable goals, small steps and explicit learning opportunities along the way.

\section{Step 5: Take the first few steps}

We implement the strategy, taking the first few steps along the chosen trajectory. At the same time we carefully monitor the effects of those first few steps. Often, the first few steps involve the use of working prototypes, rather than fully operational technologies. However, early demonstration using a prototyping

important feature of this ecosystem theory probably also applies to resource management: in the early stages of moving from the present point towards the future, it is fairly easy to introduce small shifts or to change trajectories if we find we are not on the right path, since the trajectories are not all that different. Later on, even though we are still moving towards the same set of endpoints, the distance between trajectories may be substantial, and mid-course corrections may be much more difficult and/or expensive.

\section{Step 3: Learn about how to get there}

Having decided on where to go, we should spend some time learning about how to get there: which road to take, what the terrain will be like, what equipment and supplies may be needed. Is this an ice route, a rock route or a snow route? Where will the stage camps be and how far apart? Stage camps represent interim objectives along the way to the final objective of the water resource management class: these interim objectives need to be clearly specified and understood by all parties.

As mentioned above, there may be multiple trajectories which can take us to the future we have selected. The trajectories may be longer in duration or shorter, expensive or less costly, exploitive or restorative - this choice is still ours, depending on which trajectory society considers to be the most suitable.

In mountaineering, as in water resource management, the general direction is more important than the exact endpoint of each day's efforts. The overall direction must at least be steadily upwards if we are to eventually reach the summit of the mountain. Messing around in the valley or on contour paths just because they are easy will not be useful if we do it for too long in order to avoid the tough parts of the climb.

\section{Step 4: Design/Adapt the strategy for getting to the future}

In this step, we must find an appropriate way to take actions in order to get onto a new trajectory or to make better progress on our current trajectory. This provides us with a strategy for travelling along the desired trajectory, and influences our daily business procedures which must become operational as we manage ourselves and our resources onto the chosen trajectory.

The National Water Act provides for the development of catchment management strategies, which will specify the objectives, timeframes for achieving objectives, actions to be taken and responsibilities of the various parties, including water management institutions as well as water users and stakeholders. However, we approach can be extraordinarily powerful in convincing people of the achievability of change, increasing their understanding of the vision and trajectory, overcoming resistance to change and encouraging adoption of new ways of doing things. Early prototyping is preferable since a large new, supposedly operational system is likely to scare people back across the danger zone of the change cycle.

This is the equivalent of setting out on a short journey and reaching the next stage camp. It will not help if only the specialist climbers reach the next camp - all the necessary team members must come along if the camp is to be properly established and serve as a base for the next part of the climb. Likewise, in policy implementation, all the organisations and groups which are involved need to come along to the next stage camp, and should be doing so from the beginning of the expedition, or early on in the trajectory (Fig. 3). It must be emphasised that the whole water management institution (and not only the DWAF) must take part in every stage and must reach every stage camp. This includes the various water user sectors (e.g. forestry, agriculture, tourism, suppliers of potable water), relevant research agencies and conservation bodies. Those who hang back, waiting to see where the final trajectory will lie, or those who are left out of planning steps, are likely to be left too far behind and will lack the capacity, knowledge, skills and technology to catch up with the rest of the expedition. Leadership and communication play critical roles in keeping all the team members together on the same trajectory: those who move onto different trajectories from the rest of the team may never make it to the neighbourhood of the endpoint, since the effort required to change trajectory at a later stage will be too great. Leaving some members of the "virtual" water resource management team out in the cold could seriously jeopardise success in the middle and later stages of implementation.

\section{Step 6: Going deeper: Pause, reflect and learn.}

After the first few steps along our trajectory, it is wise to pause, and reflect on the results of our implementation in order to learn (in mountaineering terms, this is where the team gathers to have tea under the camp flag at the end of each phase, and talk about all that has happened thus far). Here we want to learn about whether our actions are having (or are showing the potential to have) the desired effects: are we still on the right trajectory? Are we still headed for the right endpoint? Through monitoring, we learn more about the resources we are managing and how they respond to various actions, to see if we need to adapt our strategy in order to stay on 
this trajectory. And finally, having taken the first few steps towards our chosen future, we may now be able to see the future more clearly and need to decide whether, in this light, we should confirm, modify or refine our original decision about where we wanted to go and how we wanted to get there. So we return to the beginning of the cycle at Step 1.

This time, however, we begin the cycle with deeper knowledge and understanding, and hence an ability to do things better the next time around, including making better decisions, designing better, more detailed strategies for action, and instituting monitoring programmes which are better tailored to help us learn.

This last step of reflection and learning is absolutely critical to the expedition, and if we do not give it sufficient attention the expedition is likely to fail to progress to the next stage camp and finally to the summit. Yet how often is the step of strategic review and reflection neglected in a process of implementation? In an era and society where everything takes place in fast-forward, time for reflection is often regarded as an unnecessary luxury. Yet, making time to look back at progress to date, to instil a sense of ownership with those that participated and to jointly celebrate achievements, to look forward and appreciate the vision from a new vantage point and with new understanding and insights, can have enormous value from a psychological and communications perspective. Reflection must become a planned and explicit step in the adaptive process. It is the leader's responsibility to make sure this happens, that the right people participate in the exercise, and that all the right information is taken into account in making the decision about the next day's routes.

\section{Guiding principles for adaptive policy implementation}

An adaptive policy implementation process, while being inherently flexible, needs at the same time to be quite deliberate and measured. Because the process is based on "learn-by-doing", there is a danger that adaptations made along the way will become random, midcourse corrections made simply for the sake of making a change in order to "look busy". Any change or selection of an adapted trajectory must be made on the basis of careful consideration and reflection upon learning obtained in prior implementation. Adaptive processes are not $a d h o c$, nor are they an excuse for doing just anything, or for adopting a laissez-faire attitude. Adaptive processes should also not be seen as providing excuses to correct mistakes that arise because the original goal or plan was not clearly thought out.

\section{Three tiers of adaptive policy implementation}

The process of adaptive policy implementation (Fig. 2) occurs in parallel at different levels, nested within each other. Each time we walk through the cycle, we descend into greater detail as we move from fairly general statements about principles and policy, to quantitative, everyday operating rules for water resource systems. Three main tiers can be identified:

- The broadest tier, at the policy level, which may be national or regional policy

- The second tier, at the strategy level, which may be at water management area or catchment scale

- The third tier, at the local operational level within a catchment.

The time taken to traverse the cycle also varies between tiers. The changing of policy at the level of national constitutions may take 50 to 100 years; response of national sectoral policy, such as water policy, may operate on time scales of 20 to 30 years; strategies at water management or catchment scale can typically take 5 to 10 years to move through the cycle, while everyday operation at the local level can be adapted within a matter of weeks if necessary. An important aspect of holding the vision is keeping the larger time horizons and process functional and alive in the maelstrom of everyday activity.

\section{A role for everyone in adaptive policy implementation}

An adaptive implementation process is not all or only about changing what we do. It is about recognising the need for change, about envisioning a future and planning to get there. Most importantly though it is about implementing the plan and anchoring the vision in reality to ensure it is achieved. Different people and skills are needed for the different steps of an adaptive management cycle (Fig. 2). Since few people have the ability to do more than one of these tasks, adaptive management needs a team with many players in different positions.

The National Water Act has gone through the initial recognition and envisioning phases and we are now planning its implementation. Throughout these phases the idea "gatherers" and "planters" have been busy assembling the bricks and mortar. The "systems thinkers" have captured the ideas and integrated them into plans of what to do. Some natural "adapters" have begun building the new system, and experimenting with ideas and plans. In some instances they feed their experiences back to the "planners" in good adaptive management mode. In others the "idea gatherers" do it for them.

Soon the "finishers" will tie the loose ends together to generate line functions and reporting systems within CMAs or their precursors. Then the "anchors" will faithfully provide the services and products that successfully anchor the vision in reality.

All the while the world is changing, societal values are changing. In response the "learners" are finding ways to do it better and the "loyal heretics" start questioning the vision and whether it will be reached on the trajectory chosen. At some point they influence the "visionary" to the extent that a new or modified vision is held up and tested against societal values.

But all the while there must be "vision keepers" who watch to see that the political, economic, natural and social "crises" of the day to not side-track the team.

\section{Enabling conditions for an adaptive policy implementation process}

There are several enabling conditions which support the implementation of policy in an adaptive, stepwise process.

- We will need willing, capable adaptive organisations which can field a team with the right skills and resources, although they might not have explored this territory before. The National Water Act provides for the establishment of CMAs, and the need for these to be adaptive, learning organisations has been discussed elsewhere (Rogers et al., 2000). While CMAs will be the primary water management institutions involved in policy implementation, they will need to work in partnerships with several other types of organisation, including all spheres of government, water users and the private sector, in "virtual" institutions which share the responsibilities associated with water resources management. Keeping such virtual institutions together on the same trajectory will require trust, communication and dovetailed business processes. Taking too big a step at a time and not pausing to learn will lose team members. 
- The vision which all are trying to achieve must be explicit enough, and should be communicated to all concerned on a continuous basis, being made more explicit as more information becomes available. For example, at a water management area level, a vision for a water resource might be encapsulated in the class which is assigned to that resource, and made more explicit by the setting of quantitative resource quality objectives linked to the class. The water allocation plan provides detail on how water will be utilised, while the catchment management strategy sets out actions, responsibilities and time frames.

- An implementation plan must be in place that is robust, well understood by all, and designed to allow small steps to be taken, evolving the plan in an adaptive manner. The plan need not be final, nor does it need to contain details on each and every contingency, but explicit learning opportunities should be built into each step in order to improve the process as the plan evolves. The proposed National Water Resource Strategy (DWAF, 2002), a requirement of the National Water Act, sets out broad time scales and priorities for implementation at national level, providing at least some guidance on which particular mountains to climb first.

\section{Progress to date}

What progress is being made in establishing an enabling environment for adaptive policy implementation? To answer this question, we need to step back from the current processes and evaluate the situation from an outsider's point of view.

In terms of adaptive water management organisations or institutions, the CMA establishment process is in its early stages. It is expected that five or six CMAs may be formally established within the next two to three years (DWAF, 2002). While there is recognition of the need for collaborative partnerships with other players from within the water sector, we in South Africa have not had much practice at making such partnerships work. Responsibilities are still held along deeply divided organisational boundaries, even within single organisations such as DWAF. Significant steps must still be taken to make the boundaries more permeable and to facilitate partnerships on a basis of mutual trust and shared responsibility. The current restructuring of DWAF may advance the situation somewhat, but it will take time to change the behaviour of individuals to achieve truly collaborative partnerships amongst inherently flexible and adaptive organisations.

Efforts to communicate the vision encapsulated in the water policy have tailed off since the heady days of policy development between 1996 and 1999, when groups from across the water sector and indeed from across South African society were involved on an everyday basis in clarifying the vision and helping to draw out the details. Policy development and implementation efforts seem recently to have been drawn back within the boundaries of the lead agent, DWAF, with only limited communication reaching the "second circle" of interested members of the water sector. With reference to Fig. 3, it is worth repeating that if all parties in a collaborative partnership for policy implementation are to be fully committed and taking up their responsibilities, then they must all be on the same trajectory as the lead agent and at the same level of understanding, or their commitment will fail and their ability to participate willingly will be greatly reduced as they get left farther and farther behind.

An implementation plan, in the form of the proposed National Water Resource Strategy (DWAF, 2002), is currently in develop- ment, and was published in draft for comment during 2002. Hopefully this will contribute positively to guiding policy implementation over the next 20 years.

Perhaps the biggest challenge is changing the mindsets of people, both officials and water users, to support a truly adaptive process of policy implementation. We still have nowhere near the critical mass of people through the "danger zone" (Fig. 1) and into the positive side of the cycle of change. Many people, including those in government and water users, are firmly stuck in the discomfort zone, while only a few pioneers have moved ahead to see what lies on the other side and are now working in the discovery zone.

\section{Concluding remarks}

Is it as simple as this? The principles of strategic adaptive management are simple enough, but to implement these principles requires quite sophisticated policy tools, a supportive environment, and implementing organisations which are appropriately designed to undertake truly adaptive management. South Africa's water policy and National Water Act have provided a set of tools which will contribute greatly to achieving strategic adaptive management of water resources, and the new political dispensation provides a more supportive culture for learning and adapting. However, it will be crucial that the Catchment Management Agencies (including DWAF as the default interim CMA) are designed to be, in themselves, learning organisations which are able to foster a culture of adaptive management and truly implement the principles of adaptive management. There is much to learn, but there is much knowledge available from sources in the business world to which we as scientists and engineers may not have hitherto paid sufficient attention.

\section{References}

ALEY J, BURCH WR, CONOVER B and FIELD D (eds.) (1999) Ecosystem Management: Adaptive Strategies for Natural Resource Organizations in the Twenty-First Century. Taylor \& Francis, London.

BROCK LR and SALERNO MA (1998) The Secret to Getting Through Life's Difficult Changes. Bridge Builder Media, Washington DC/ Durban RSA.

DWAF (1997) White Paper on a National Water Policy for South Africa. Department of Water Affairs and Forestry, Pretoria, South Africa.

DWAF (2002) Proposed National Water Resource Strategy. Department of Water Affairs and Forestry, Pretoria, South Africa.

FULMER WE (2000) Shaping the Adaptive Organization: Landscapes, Learning, and Leadership in Volatile Times. AMACOM, New York.

HAECKEL SH (1999) Adaptive Enterprise - Creating and Leading Sense-and-Respond Organizations. Harvard Business School Press, Boston.

PIETERS GW (1999) The Ever-Changing Organization: Creating the Capacity for Continuous Change, Learning and Improvement. CRC Press - St Lucie Press

ROGERS KH and BESTBIER R (1997) Development of a Protocol for the Definition of the Desired State of Riverine Systems in South Africa. Department of Environmental Affairs and Tourism, Pretoria.

ROGERS KH, ROUX D and BIGGS H (2000) Challenges for catchment management agencies: Lessons from bureaucracies, business and resource management. Water SA 26 (4) 505-511.

ROLING NG and WAGEMAKERS MAE (eds.) (1998) Facilitating Agriculture: Participating Learning and Management in Times of Environmental Uncertainty. Cambridge University Press, Boston.

WALTERS CJ (1997) Challenges in adaptive management of riparian and coastal ecosystems. Conservation Ecology. [on-line] 1(2)1. Available from the Internet. URL: http://www.consecol.org/vol1/iss2/art1. 